\title{
On the early-time chemistry in dark clouds
}

\author{
H. S. Liszt \\ National Radio Astronomy Observatory, 520 Edgemont Road, Charlottesville, VA, USA 22903-2475, USA \\ e-mail: hliszt@nrao.edu
}

Received 12 August 2009 / Accepted 13 October 2009

\begin{abstract}
Context. Agreement between observed and calculated abundances of some molecules in dark clouds is improved when the model gas retains some memory of the assumed initial conditions.

Aims. We assess the relevance of commonly-assumed intitial conditions giving rise to so-called early-time chemistry in models of dark cloud chemistry.

Methods. Initial conditions for models of dark cloud chemistry are compared with conditions known to exist in the $\mathrm{H}_{2}$-bearing diffuse gas whose evolution they are intended to represent.

Results. The initial conditions assumed for dark cloud chemistry differ in at least five important regards from those of $\mathrm{H}_{2}$-bearing diffuse gas. Models typically assume: absolute purity of $\mathrm{H}_{2}$; absence of polycyclic aromatic hydrocarbons; two times smaller gasphase abundance of $\mathrm{C}, \mathrm{N}$, and $\mathrm{O}$; near-absence of sulfur and some other easily-ionized metals; total absence of the molecules which accompany the presence of $\mathrm{H}_{2}$ in diffuse gas, for instance, $\mathrm{OH}, \mathrm{CH}, \mathrm{C}_{2} \mathrm{H}, \mathrm{HCO}^{+}$.

Conclusions. Initial conditions commonly assumed for dark cloud chemical models should be reconsidered and mechanisms for the chemical evolution from diffuse to dark gas should be included in model calculations.
\end{abstract}

Key words. astrochemistry - ISM: molecules

\section{Introduction}

Comprehensive models of dark cloud chemistry typically calculate time-dependent abundances, starting from initial conditions that are supposed to represent diffuse gas which has suddenly made the transition to a colder, much denser and darker state (Graedel et al. 1982; Leung et al. 1984; Hasegawa et al. 1992; Ruffle \& Herbst 2001; Garrod et al. 2007; Quan et al. 2008; Bryans et al. 2009). One such set of initial conditions is reproduced in Table 1 here. The model gas is initially devoid of trace molecules and has a high concentration of $\mathrm{C}^{+}$and electrons, but under these conditions the calculated abundances of some species agree with observations best at the so-called early times, i.e. within perhaps $5 \times 10^{5} \mathrm{yr}$, well before chemical equilibrium is established. For instance, Cordiner et al. (2007) discuss molecular anion abundances at early times and Quan et al. (2008) show that high abundances of $\mathrm{O}_{2}$ are difficult to suppress in pure gas phase models once $\mathrm{OH}$ becomes abundant at $3 \times 10^{5} \mathrm{yr}$. Reliance on these initial conditions to improve agreement between calculations and observations has been criticized by Gerin et al. (2003).

Here we discuss the assumed intitial conditions that give rise to the present ideas of early-time chemistry in dark cloud chemical models. We argue that these starting conditions, which originated 25-30 years ago in a more primitive epoch in our understanding of the diffuse interstellar medium (ISM), cannot be supported. They should be replaced by a more appropriate set of initial assumptions and supplemented, if necessary, with a physically or at least empirically-based description of how certain supposed important distinctions between diffuse and dark gas especially the free gas-phase elemental abudances - could have arisen, if indeed they are not entirely artificial.
In Sect. 2 we discuss five important ways in which the initial assumptions of dark cloud chemistry are subject to question. Section 3 presents a brief summary and discussion of how to bridge the gap between descriptions of the diffuse and dark gas.

\section{Conditions in $\mathrm{H}_{2}$-bearing diffuse gas vs. those of "early-times" in dark cloud chemical models}

The origins of the presently-adopted initial conditions for dark cloud chemical models date back at least to the work of Graedel et al. (1982) who referenced the ion and atomic elemental abundances tabulated by Mitchell et al. (1978) for the archtypical diffuse cloud at $A_{\mathrm{V}}=1 \mathrm{mag}$ toward $\zeta$ Oph. Some version of these conditions has subsequently been adopted in many later and recent models, as noted in very summary fashion in the Introduction. One very recent version is quoted in Table 1 here.

As seen in Table 1, all of the H-nuclei are assumed to be initially in $\mathrm{H}_{2}$, the only form of gas-phase carbon is $\mathrm{C}^{+}$, and various heavier metals are also present as the first ion. Oxygen and nitrogen are neutral, conditions which are roughly appropriate for diffuse gas (in which small abundances of the neutral carbon and other metals are present) but the abundances of the metals relative to hydrogen are below, perhaps very much below, those of the diffuse interstellar ISM (as discussed subsequently). Note also that the abundance of ${ }^{4} \mathrm{He}$ is the primordial value, which underestimates the local galactic disk abundance by some $30 \%$, see Balser (2006).

The initial conditions chosen for the dark cloud chemistry differ in at least five important regards from those which obtain in diffuse gas. In the starting conditions for the dense gas model: all of the $\mathrm{H}$-nuclei are in $\mathrm{H}_{2}$; the PAH which heat diffuse gas and control the atomic ionization (Wolfire et al. 1995a; 
Table 1. Initial nonzero fractional abundances ${ }^{1}$.

\begin{tabular}{lcc}
\hline \hline Species & L134N & TMC-1 \\
\hline $\mathrm{He}$ & $6.00 \times 10^{-2}$ & $6.00 \times 10^{-2}$ \\
$\mathrm{~N}$ & $2.14 \times 10^{-5}$ & $2.14 \times 10^{-5}$ \\
$\mathrm{O}$ & $1.76 \times 10^{-4}$ & $6.10 \times 10^{-5}$ \\
$\mathrm{H}_{2}$ & $5.00 \times 10^{-1}$ & $5.00 \times 10^{-1}$ \\
$\mathrm{C}^{+}$ & $7.30 \times 10^{-5}$ & $7.30 \times 10^{-5}$ \\
$\mathrm{~S}^{+}$ & $8.00 \times 10^{-8}$ & $8.00 \times 10^{-8}$ \\
$\mathrm{Si}^{+}$ & $8.00 \times 10^{-9}$ & $8.00 \times 10^{-9}$ \\
$\mathrm{Fe}^{+}$ & $3.00 \times 10^{-9}$ & $3.00 \times 10^{-9}$ \\
$\mathrm{Na}^{+}$ & $2.00 \times 10^{-9}$ & $2.00 \times 10^{-9}$ \\
$\mathrm{Mg}^{+}$ & $7.00 \times 10^{-9}$ & $7.00 \times 10^{-9}$ \\
$\mathrm{P}^{+}$ & $3.00 \times 10^{-9}$ & $3.00 \times 10^{-9}$ \\
$\mathrm{Cl}^{+}$ & $4.00 \times 10^{-9}$ & $4.00 \times 10^{-9}$ \\
$\mathrm{~F}^{+}$ & $6.69 \times 10^{-9}$ & $6.69 \times 10^{-9}$ \\
\hline
\end{tabular}

${ }^{1}$ Reproduced from Quan et al. (2008).

Liszt 2003; Tielens 2008) are absent; carbon, oxygen and nitrogen are two times more strongly depleted from the gas; the heavier metals with low ionization potentials, especially sulfur, are depleted by additional factors of $10^{3}$ or more; except for $\mathrm{H}_{2}$, no molecules are present and even many important neutral atoms $\left(\mathrm{H}^{0}, \mathrm{C}^{0}\right)$ are missing.

Each of these differences is discussed individually in the following sections. The point is that the initial conditions assumed to apply for the dense gas chemical models (and certainly not just that work from which the entries in Table 1 were taken) are neither self-consistent nor are they obvious consequences of the conditions in which the gas might have existed prior to becoming dark and dense.

\subsection{The $H^{0} / H_{2}$ ratio}

$\mathrm{H}_{2}$ is easily detected in diffuse clouds (Savage et al. 1977; Rachford et al. 2002) and even typical "Spitzer" H I clouds with $N(\mathrm{H})=3 \times 10^{20} \mathrm{~cm}^{-2}, n(\mathrm{H})=30 \mathrm{~cm}^{-3}$ are expected to be substantially molecular in their innermost regions, with some $25 \%$ of the H-nuclei in $\mathrm{H}_{2}$ (Liszt 2007b). Nonetheless, the very high (but not absolute) degrees of purity of $\mathrm{H}_{2}$ which actually obtain in many dark clouds, where $n\left(\mathrm{H}^{0}\right) / n(\mathrm{H}) \ll 0.01$, require long times to achieve, as noted by Goldsmith (2007). This is shown in Figs. 1 and 2 here for gas which at $t=0$ suddenly becomes dense (at the densities $n(\mathrm{H})$ of H-nuclei indicated), cold $\left(T_{\mathrm{K}}=12 \mathrm{~K}\right)$ and dark (i.e. is deprived of all $\mathrm{H}_{2}$-dissociating $u v$ photons) so that the only means of dissociating $\mathrm{H}_{2}$ is cosmic rays; the calculations are shown for low and higher values of $\zeta_{\mathrm{H}}$, the rate per $\mathrm{H}$-nucleus, as indicated. In this case the evolution is given by an analytic solution, Eq. (2) of Liszt (2006). The solutions here differ (only) parametrically from the curves shown by Goldsmith \& $\mathrm{Li}$ (2005), whose $\mathrm{H}_{2}$-formation rate was $60 \%$ that adopted here, so that the evolution in our figures is that much more rapid.

Figure 1 shows the evolution of a relatively dense gas for various initial purities of $\mathrm{H}_{2}$; evolution of the $\mathrm{H}_{2}$ fraction continues for $0.3-1.0 \times 10^{6} \mathrm{yr}$, which roughly coincides with the epoch described as "early-time" in chemical models. As indicated in Fig. 2, the equilibrium time decreases as $1 / n(\mathrm{H})$, so that only if $n(\mathrm{H}) \gg 10^{4} \mathrm{~cm}^{-3}$ might it be expected that the $\mathrm{H}_{2}$-fraction could evolve substantially faster than the early-time chemistry as a whole. Such high densities are not generaly characteristic of dark clouds or the model calculations.

This discussion implies that the $\mathrm{H}_{2}$ fraction should be allowed to evolve from a state with substantial initial amounts of

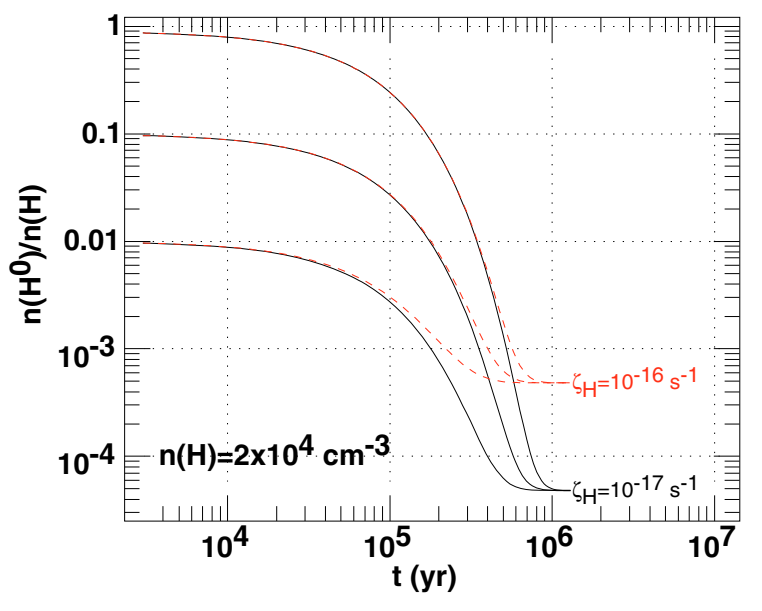

Fig. 1. Evolution of the atomic gas fraction $n\left(\mathrm{H}^{0}\right) / n(\mathrm{H})$ in a dark gas of total density of H-nuclei $n(\mathrm{H})=2 \times 10^{4} \mathrm{~cm}^{-3}$ at $T_{\mathrm{K}}=12 \mathrm{~K}$, for two values of the cosmic ray ionization rate $\zeta_{\mathrm{H}}$ per $\mathrm{H}$-nucleus and three initial values $n\left(\mathrm{H}^{0}\right) / n(\mathrm{H})=1,0.1$, and 0.01. See Sect. 2.1 of the text.

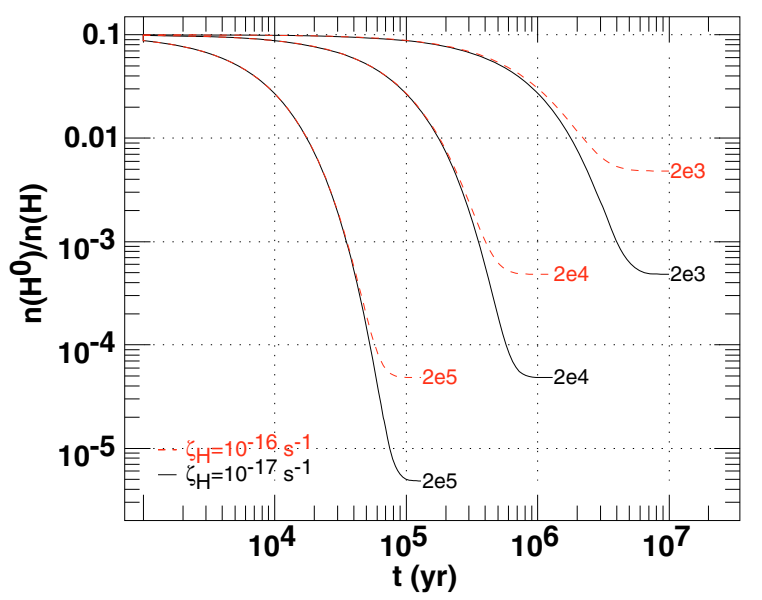

Fig. 2. Evolution of the atomic gas fraction $n\left(\mathrm{H}^{0}\right) / n(\mathrm{H})$ in dark gases of total density $n(\mathrm{H})=2 \times 10^{3} \mathrm{~cm}^{-3}, 2 \times 10^{4} \mathrm{~cm}^{-3}$ and $2 \times 10^{5} \mathrm{~cm}^{-3}$ at $T_{\mathrm{K}}=12 \mathrm{~K}$, for two values of the cosmic ray ionization rate $\zeta_{\mathrm{H}}$ per $\mathrm{H}$-nucleus as in Fig. 1. At each density the higher-lying (red, dashed) curve represents the larger value of $\zeta_{\mathrm{H}}$. See Sect. 2.1 of the text.

atomic hydrogen - much more than at equilibrium: to do otherwise (as now) is to assume that the gas has already undergone a long period of evolution in its dense dark state even before the calculation has begun, a contradiction. The abundance of atomic hydrogen is important for the chemistry in its own right. As shown by Rawlings et al. (2002), a high initial H-fraction strongly depresses the early-time abundances of most molecules, although a few, most notaby $\mathrm{OH}$, are somewhat increased. As recently stressed by Flower et al. (2007) the abundance of molecular anions is controlled by the ratio $n\left(\mathrm{e}^{-}\right) / n\left(\mathrm{H}^{0}\right)$. In current models $n\left(\mathrm{H}^{0}\right) / n(\mathrm{H})$ increases with time, while physically it should be expected to decrease as in Figs. 1, 2.

\section{2. $\mathrm{PAH}$ and the initial charge state}

The assumption in chemical models that carbon is initially present solely as $\mathrm{C}^{+}$is a somewhat extreme version of the circumstances observed in diffuse gas where the carbon indeed exists predominantly as $\mathrm{C}^{+}$even when substantial quantities of CO exist in the gas (Liszt 2007a), but typical observed ratios of $\mathrm{C}^{0} / \mathrm{C}^{+}$are in the range $0.001-0.01$ (as toward $\zeta \mathrm{Oph}$ ) in 
$\mathrm{H}_{2}$-bearing diffuse gas. Moreover, electron recombination of the $\mathrm{C}^{+}$in the dense dark chemically-modeled gas is relatively rapid anyway, but in diffuse gas the neutralization of atomic ions actually occurs even faster during their interaction with PAH (Wolfire et al. 1995b, 2003; Liszt 2003; Tielens 2008). The importance of such grain-assisted neutralization is moderated at lower temperatures compared to direct electron recombination, because the rate of the former varies as $T_{\mathrm{K}}{ }^{1 / 2}$ and that of the latter typically as $T_{\mathrm{K}}{ }^{-1 / 2}$ or faster, but this only compensates for a portion of the disparity in rates overall. Bryans et al. (2009) recently noted that the electron recombination rates of atomic ions have been underestimated by factors of order three owing to the neglect of dielectronic processes, further narrowing the gap between electron and grain-assisted recombination rates at low temperatures. Bryans et al. (2009) show that increased $\mathrm{C}^{+}$ recombination rates have a pronounced effect on a coupled-gas grain chemistry in dense clouds because of the higher density of neutral carbon.

The point is that if the PAH persist into the dense dark state for even $10^{4} \mathrm{yr}$, recombination of carbon and the other atomic ions will occur within this period, i.e. almost before the chemical calculation begins and much more quickly than is now calculated using only electron recombination. Although the effects of PAH were originally considered for both diffuse and dense gas (Lepp et al. 1988; Lepp \& Dalgarno 1988) their presence in dense dark gas was subsequently deprecated, presumably on the grounds that they would coagulate into larger particles; this process should properly be considered as part of the early chemical evolution. PAH have recently been reconsidered in dense gas by Wakelam \& Herbst (2008) who showed that the apparent need for an ad hoc assumption of small sulfur abundances in dense gas (the so-called low metals case) may be eliminated. This is discussed subsequently.

\subsection{Depletion of carbon, oxygen and nitrogen}

As shown in Table 1, the initial abundances of carbon, nitrogen and oxygen are about two times smaller in chemical calculations of dense gas than is observed in the mean in the diffuse interstellar medium. For instance, the ratio of carbon to hydrogen nuclei in Table 1 is $n\left(\mathrm{C}^{+}\right) / 2 N\left(\mathrm{H}_{2}\right)=0.73 \times 10^{-4}$, a factor of two below the mean observed in diffuse gas, $N\left(\mathrm{C}^{+}\right) / N(\mathrm{H})=1.4-1.6 \times 10^{-4}$ (Cardelli et al. 1996; Sofia et al. 2004). Because so much of the free gas phase carbon eventually finds its way into $\mathrm{CO}$, the final $\mathrm{CO} / \mathrm{H}_{2}$ ratio will generally be too large in the models if the larger CNO depletions do not obtain somehow. However, it is not consistent to assume that such strong depletion is already present in the gas at the same moment that the carbon is ionized: either the free carbon abundance should evolve or an alternative, physically-based explanation for the $\mathrm{CO}$ abundance should be found.

\subsection{The low-metals case}

As shown in Table 1, the initial abundances of metals heavier than oxygen are generally assumed to be very small. While it is true that many of the easily-ionized metals heavier than oxygen are strongly depleted in the diffuse gas - although hardly by such large factors as in Table 1 - there are also some very conspicuous exceptions (Savage \& Sembach 1996). This is especially important in the case of sulfur, the most abundant of all of these with $([\mathrm{S}] /[\mathrm{H}])_{\text {Sun }}=-4.76$ (Lodders 2003). Sulfur is not generally believed to be depleted in the nearby interstellar gas, in which case its appropriate starting abundance is somewhere between the Solar value and the slightly larger values (0.1-0.2 dex) measured in local OB stars (Daflon et al. 2003). Note that Jenkins (2009) has recently raised the possibility that there is some depletion and variability in the gas-phase sulfur abundance.

The marriage of low metal abundances and dark cloud chemistry is a complicated one made for the convenience of modellers, owing to the effects of metal atoms and their ions on the overall charge balance (Oppenheimer \& Dalgarno 1974); if the low ionization potential metals are too abundant in most present chemical models they will suppress the formation of ions such as $\mathrm{H}_{3}{ }^{+}$and $\mathrm{HCO}^{+}$, squelching the ion-molecule chemistry generally. Conversely, assumption of a very low sulfur abundance can create other problems when attempting to explain the observed abundances of a wide variety of sulfur-bearing molecules. For instance, the required abundances of $\mathrm{OH}$ may be much higher than are observed. Goicoechea et al. (2006) have argued that molecular abundances in the dense Horsehead PDR are best reproduced without strong sulfur depletion.

Beyond the seeming exigencies of the chemistry, there is really no justification for assuming low sulfur abundances in dense dark gas, even more so during the early times when the material is supposed to have retained some memory of the diffuse state. As noted above, Wakelam \& Herbst (2008) have recently revived consideration of the effects of PAH in dense gas, and they note that the low metal case is not needed to preserve the efficacy of the ion-molecule chemistry when PAH are present. Another alternative may be consideration of small-molecule (as opposed to $\mathrm{PAH}$ ) anions and there may be yet other means of bearing charge in the presence of high metal abundances without ruining the ion-molecule chemistry.

\subsection{The absence of trace molecules inherited from the diffuse gas}

The presence of metal-bearing trace diatomic molecules $\mathrm{CH}$, $\mathrm{CH}^{+}$and $\mathrm{CN}$ in diffuse gas has been known for some 70 years (for a recent review see Snow \& McCall 2006) but only recently has there been a real appreciation of the wide range of species which are present in diffuse gas. Moreover it has been apparent since the Copernicus observations of $\mathrm{H}_{2}$ (Savage et al. 1977) that the relative abundances $N(\mathrm{CH}) / N\left(\mathrm{H}_{2}\right)$ are comparable in TMC-1 and diffuse clouds, and this can now be seen to apply to other (not all) species, especially $\mathrm{OH}, \mathrm{HCO}^{+}$and $\mathrm{C}_{2} \mathrm{H}$, even when $A_{\mathrm{V}} \lesssim 1$ mag ((Snow \& McCall 2006; Liszt et al. 2006). In a calculation such as that of Quan et al. (2008) the early-time chemistry is largely defined by the interval required for the $\mathrm{OH}$ abundance, initially zero, to rise to the values seen in diffuse clouds, $N(\mathrm{OH}) / N\left(\mathrm{H}_{2}\right)=10^{-7}$.

Perhaps most strikingly, the relative abundance $n\left(\mathrm{H}_{3}{ }^{+}\right) / n(\mathrm{H})$ has been found to be higher in diffuse clouds (Geballe et al. 1999), presumably owing to a higher cosmic-ray ionization rate (Indriolo et al. 2009). Because $\mathrm{H}_{3}{ }^{+}$is the driver of dark cloud chemistry, accounting for its initial abundance is of obvious importance for chemical models: although it is a somewhat higherorder process, formation of $\mathrm{H}_{3}{ }^{+}$happens on the same time scale as that of $\mathrm{H}_{2}$ as a whole (Liszt 2007b).

The absence of these inherited seed molecules from the initial conditions which presuppose a pure- $\mathrm{H}_{2}$ gas is difficult to understand, but amending this oversight raises a host of complications. For instance, the molecular inventory of diffuse gas is not nearly as complete as that of the models, so which species would be included? But perhaps more importantly, if the abundances of molecules in diffuse gas are comparable to those in TMC-1, what 
is the actual role of time-dependent chemistry at early times in the evolution from diffuse to dark conditions?

\section{Summary and discussion}

The initial conditions that are typically assumed to apply in timedependent models of dark cloud chemistry are holdovers from an earlier era of understanding of the diffuse interstellar medium, especially its $\mathrm{H}_{2}$-bearing portions. The roster of trace molecules known to exist with high abundances in diffuse clouds - comparable to those in TMC-1 - has expanded greatly, the role of $\mathrm{PAH}$ has been elaborated, and such knowledge should be incorporated into the assumed starting conditions of dark cloud models. Moreover, elemental abundances in the diffuse gas have been more firmly established, highlighting the fact that the assumed starting abundances of carbon, nitrogen, oxygen and sulfur in chemical models have been tailored ad hoc to suit the exigencies of the reaction schemes which are employed. Of course, without gas-grain coupling the initial free gas-phase elemental abundances will persist throughout the calculations.

Some minor suggestions for bridging the gap between diffuse and dark conditions are given in Sect. 2 here. Perhaps the most straightforward is to assume a non-trivial initial abundance of $\mathrm{H}^{0}$, given the times which are required for evolution of the $\mathrm{H}_{2}$ fraction. The fate of other constituents of the diffuse gas, for instance the PAH, is more ineffable; nonetheless, critically important aspects of the cloud physics, in particular the charge balance and recombination of $\mathrm{C}^{+}$, and the utility of assuming high depletions of sulfur and other easily-ionized metals, depend on the evolution of the atomic gas fraction and the PAH abundance.

Perhaps most problematic are the high abundances observed for $\mathrm{H}_{3}{ }^{+}$and the metal-bearing trace molecule such as $\mathrm{CH}, \mathrm{OH}$ and $\mathrm{HCO}^{+}$in diffuse gas; one must ask whether including them in the starting conditions would not be a repudiation of the entire notion of a distinct early-time chemistry. Although the most promising schemes for producing high molecular abundances in diffuse gas can be quenched at high mean density (Godard et al. 2009) it is hard to credit the idea that the trace molecules simply disappear and must be recreated from scratch. If that were the case, it would be possible to observe fairly dark $\left(A_{\mathrm{V}}>1 \mathrm{mag}\right)$, largely molecular gas with anomalously small abundances of even such simple and easily-observed species as $\mathrm{CH}$ and $\mathrm{OH}$. That such conditions have never been observed seems a strong argument for continuity of the trace molecule abundances during the early chemical evolution. In any case, the discussion here highlights the importance of measuring elemental abundances and physical conditions in the so-called translucent clouds with $1 \lesssim A_{\mathrm{V}} \lesssim 3$ mag which may embody the intermediate state between diffuse and dark gas.
Acknowledgements. The National Radio Astronomy Observatory is operated by Associated Universites, Inc. under a cooperative agreement with the US National Science Foundation. I am grateful to Dr. Lucy Ziurys for a stimulating discussion of this subject and to the referee for his comments.

\section{References}

Balser, D. S. 2006, AJ, 132, 2326

Bryans, P., Kreckel, H., Roueff, E., Wakelam, V., \& Savin, D. W. 2009, ApJ, 694, 286

Cardelli, J. A., Meyer, D. M., Jura, M., \& Savage, B. D. 1996, ApJ, 467, 334

Cordiner, M. A., Millar, T. J., Herbst, E., Chuimin, R. N., \& Walsh, C. 2007, in Molecules in Space and Laboratory

Daflon, S., Cunha, K., Smith, V. V., \& Butler, K. 2003, A\&A, 399, 525

Flower, D. R., Pineau Des Forêts, G., \& Walmsley, C. M. 2007, A\&A, 474, 923

Garrod, R. T., Wakelam, V., \& Herbst, E. 2007, A\&A, 467, 1103

Geballe, T. R., McCall, B. J., Hinkle, K. H., \& Oka, T. 1999, ApJ, 510, 251

Gerin, M., Fossé, D., \& Roueff, E. 2003, in SFChem 2002: Chemistry as a Diagnostic of Star Formation, ed. C. L. Curry, \& M. Fich, 81

Godard, B., Falgarone, E., \& Pineau Des Forêts, G. 2009, A\&A, 495, 847

Goicoechea, J. R., Pety, J., Gerin, M., et al. 2006, A\&A, 456, 565

Goldsmith, P. F. 2007, in Molecules in Space and Laboratory, ed. J. L. Lemaire, \& F. Combes, 160

Goldsmith, P. F., \& Li, D. 2005, ApJ, 622, 938

Graedel, T. E., Langer, W. D., \& Frerking, M. A. 1982, ApJS, 48, 321

Hasegawa, T. I., Herbst, E., \& Leung, C. M. 1992, ApJS, 82, 167

Indriolo, N., Fields, B. D., \& McCall, B. J. 2009, ApJ, 694, 257

Jenkins, E. B. 2009, ApJ, 700, 1299

Lepp, S., \& Dalgarno, A. 1988, ApJ, 324, 553

Lepp, S., Dalgarno, A., van Dishoeck, E. F., \& Black, J. H. 1988, ApJ, 329, 418 Leung, C. M., Herbst, E., \& Huebner, W. F. 1984, ApJS, 56, 231

Liszt, H. 2003, A\&A, 398, 621

Liszt, H. S. 2006, A\&A, 458, 507

Liszt, H. S. 2007a, A\&A, 476, 291

Liszt, H. S. 2007b, A\&A, 461, 205

Liszt, H., Lucas, R., \& Pety, J. 2006, A\&A, 448, 253

Lodders, K. 2003, ApJ, 591, 1220

Mitchell, G. F., Kuntz, P. J., \& Ginsburg, J. L. 1978, ApJS, 38, 39

Oppenheimer, M., \& Dalgarno, A. 1974, ApJ, 192, 29

Quan, D., Herbst, E., Millar, T. J., et al. 2008, ApJ, 681, 1318

Rachford, B. L., Snow, T. P., Tumlinson, J., et al. 2002, ApJ, 577, 221

Rawlings, J. M. C., Hartquist, T. W., Williams, D. A., \& Falle, S. A. E. G. 2002, A\&A, 391, 681

Ruffle, D. P., \& Herbst, E. 2001, MNRAS, 322, 770

Savage, B. D., \& Sembach, K. R. 1996, ARA\&A, 34, 279

Savage, B. D., Drake, J. F., Budich, W., \& Bohlin, R. C. 1977, ApJ, 216, 291

Snow, T. P., \& McCall, B. J. 2006, ARA\&A, 44, 367

Sofia, U. J., Lauroesch, J. T., Meyer, D. M., \& Cartledge, S. I. B. 2004, ApJ, 605, 272

Tielens, A. G. G. M. 2008, ARA\&A, 46, 289

Wakelam, V., \& Herbst, E. 2008, ApJ, 680, 371

Wolfire, M. G., Hollenbach, D., McKee, C. F., Tielens, A. G. G. M., \& Bakes, E. L. O. 1995a, ApJ, 443, 152

Wolfire, M. G., McKee, C. F., Hollenbach, D., \& Tielens, A. G. G. M. 1995b, ApJ, 453, 673

Wolfire, M. G., McKee, C. F., Hollenbach, D., \& Tielens, A. G. G. M. 2003, ApJ, 587,278 